Jonathan Stewart, BS

Philadelphia College of Osteopathic

Medicine, Philadelphia, PA
Grace U. Salacup, MD

Albert Einstein Medical Center, Philadelphia, PA
Kevin Bryan Uy Lo, MD

Albert Einstein Medical Center,

Philadelphia, PA

\title{
A patient with breast cancer and a rash on her hands and feet
}
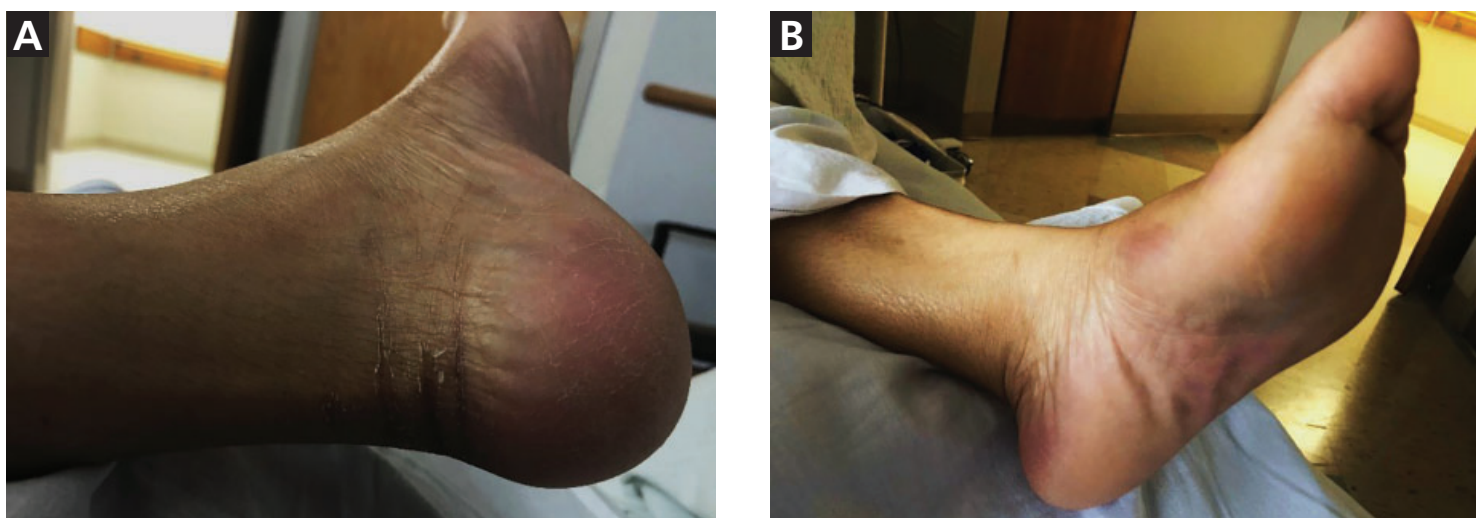

Figure 1. Erythema and skin desquamation on the heel $(A)$, and patchy erythema over the plantar and lateral foot (B).

Treatment

strategy:

chemotherapy

interruption,

longer intervals

between

treatments, and

dose reduction 56-year-old woman with invasive triple receptor-positive ductal carcinoma of the breast presented with persistent vomiting, diarrhea, and abdominal pain. She had completed her third cycle of docetaxel, carboplatin, trastuzumab, and pertuzumab chemotherapy 3 days earlier.

She was afebrile, her pulse rate was 126 beats per minute, and her blood pressure was 121/66 mm Hg. Her abdomen was diffusely tender without rebound or guarding.

Laboratory testing revealed a white blood cell count of $4.3 \times 10^{9} / \mathrm{L}$ (reference range 4.0-11.0 $\times$ $10^{9} / \mathrm{L}$ ) and normal serum electrolyte levels.

She was admitted for chemotherapy-related intractable vomiting and diarrhea and was started on intravenous fluids and symptomatic management.

On hospital day 4, she reported burning pain in her left foot. Examination revealed erythema over the plantar surface of the left foot, with pain worsened by palpation and ambuladoi:10.3949/ccjm.88a.20020 tion. She remained afebrile and was started on cefazolin for empiric treatment of cellulitis in the setting of an immunosuppressed state.

Over the next 2 days, the pain progressed to include both hands and feet. Examination showed erythema involving the plantar, lateral, and calcaneal regions of both feet with mild skin desquamation (Figure 1). Also noted were erythema of the palmar surface of the hands and onychomelanosis (Figure 2). Antibiotics were stopped. The oncology service deemed these symptoms as hand-foot syndrome secondary to docetaxel treatment. The patient's gastrointestinal symptoms improved, and 2 days after the antibiotics were stopped, she was discharged with emollients for her hands and feet.

\section{HAND-FOOT SYNDROME}

Hand-foot syndrome is a cutaneous adverse effect of cytotoxic chemotherapy. It can present as palmar-plantar dysesthesia manifested as tingling and burning pain followed by an erythematous rash, dry skin, desquamation, or 
ulceration of the palms of the hands and soles of the feet 2 to 12 days after chemotherapy. ${ }^{1}$

Pathophysiologic mechanisms include rapidly dividing skin cells, gravitational forces, anatomic abundance, and dilation of blood vessels in these areas, as well as increased drug concentration in the eccrine glands of the palms and soles., ${ }^{1,2}$

Chemotherapy agents associated with hand-foot syndrome include 5-fluorouracil, liposomal doxorubicin, docetaxel, and capecitabine. ${ }^{1}$ Docetaxel in particular has been reported to cause the syndrome when used as a single chemotherapeutic agent.,4

The risk of developing hand-foot syndrome appears to depend on peak drug concentration and total cumulative dose. Therefore, a major treatment strategy is to interrupt the chemotherapy, lengthen the interval between treatments, and reduce the dose. Other options include cold compresses, leg elevation, topical or systemic steroids, creams, and pyridoxine. ${ }^{1,2}$

Clinicians should keep in mind that handfoot syndrome can mimic other common conditions such as erythromelalgia, chemotherapy-induced Raynaud phenomenon, erythema multiforme, infection (cellulitis, erysipelas), and other drug reactions. ${ }^{5}$

Although the syndrome is self-limiting, it can have a significant negative impact on quality of life due to pain and limitation in daily activities. ${ }^{2}$ Because it is directly related to dose or duration of drug administration and can recur with subsequent exposure, chemotherapy dose modifications may be needed. ${ }^{2}$

\section{TAKE-HOME POINTS}

In patients on chemotherapy, clinicians need to be aware of hand-foot syndrome in the differential diagnosis of extremity pain and erythema, its implications for quality of life, and available treatment options. Hand-foot syndrome is self-limiting and can be managed supportively together with chemotherapy in-

\section{REFERENCES}

1. Webster-Gandy JD, How C, Harrold K. Palmar-plantar erythrodysesthesia (PPE): a literature review with commentary on experience in a cancer centre. Eur J Oncol Nurs 2007; 11(3):238-246. doi:10.1016/j.ejon.2006.10.004

2. Farr KP, Safwat A. Palmar-plantar erythrodysesthesia associated with chemotherapy and its treatment. Case Rep Oncol 2011; 4(1):229-235. doi:10.1159/000327767

3. Katoh M, Kadota M, Nishimura Y. A case of docetaxel-induced erythrodysesthesia. J Dermatol 2004; 31(5):403-406.
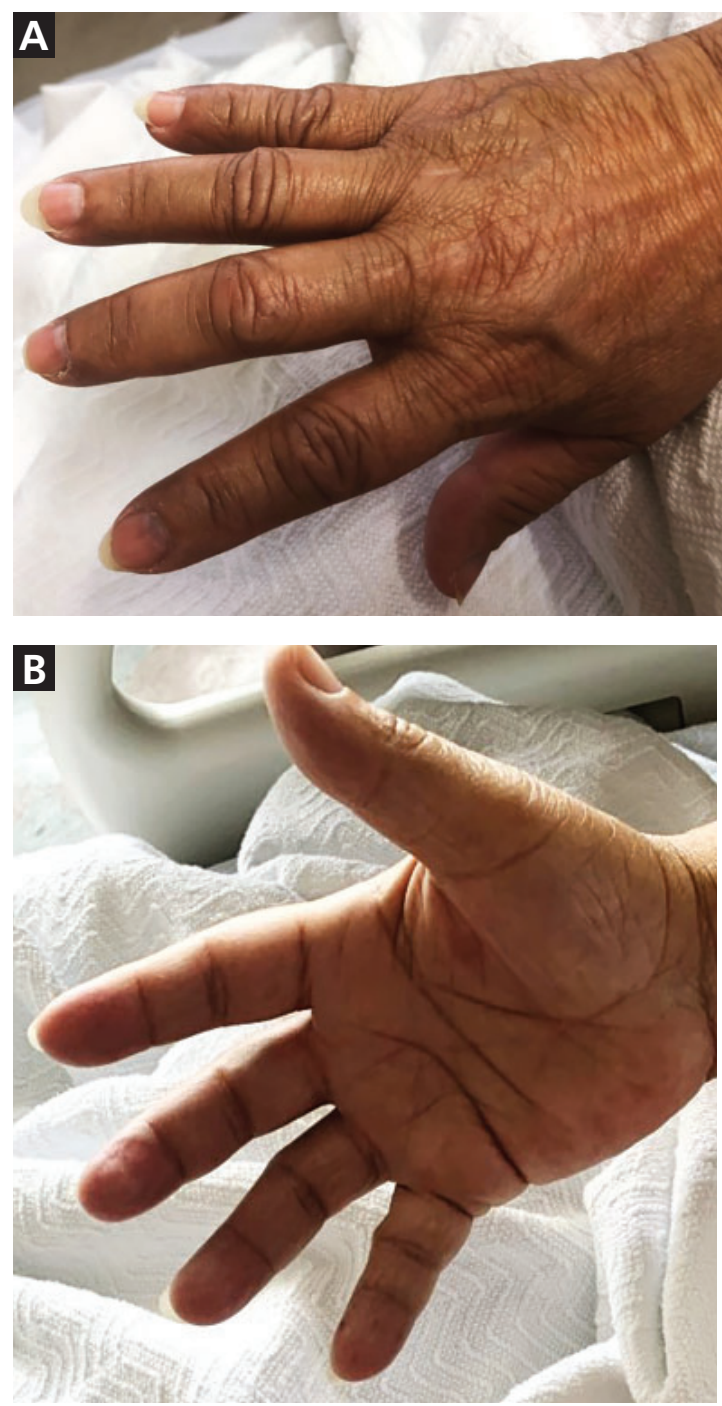

Figure 2. Note the violaceous nailbed of the second digit (onychomelanosis) (A), with patchy erythema prominent over the hypothenar eminence $(B)$.

terruption, interval lengthening, and dose reduction.

\section{DISCLOSURES}

The authors report no relevant financial relationships which, in the context of their contributions, could be perceived as a potential conflict of interest. doi:10.1111/j.1346-8138.2004.tb00692.x

4. Zimmerman GC, Keeling JH, Burris HA, et al. Acute cutaneous reactions to docetaxel, a new chemotherapeutic agent. Arch Dermatol 1995; 131(2):202-206. pmid:7857119

5. Qiao J, Fang $\mathbf{H}$. Hand-foot syndrome related to chemotherapy. CMAJ 2012; 184(15):E818. doi:10.1503/cmaj.111309

Address: Kevin Bryan Uy Lo, MD, Albert Einstein Medical Center, 5501 Old York Road, Philadelphia, PA 19141; lokevinmd@gmail.com 